\title{
Association of a Low Geriatric Nutritional Risk Index with Higher Adverse Outcome in the Elderly Patients with Fall Injuries: Analysis of a Propensity Score-Matched Population
}

This article was published in the following Dove Press journal:

Risk Management and Healthcare Policy

\author{
Szu-Wei Huang' \\ Shih-Min Yin (ID ${ }^{2}$ \\ Ching-Hua Hsieh (D) ${ }^{3}$ \\ 'Department of Obstetrics and \\ Gynecology, Kaohsiung Chang Gung \\ Memorial Hospital, Chang Gung \\ University College of Medicine, \\ Kaohsiung, 8330I, Taiwan; ${ }^{2}$ Department \\ of General Surgery, Kaohsiung Chang \\ Gung Memorial Hospital, Chang Gung \\ University College of Medicine, \\ Kaohsiung, 8330I, Taiwan; ${ }^{3}$ Department \\ of Plastic Surgery, Kaohsiung Chang Gung \\ Memorial Hospital, Chang Gung \\ University College of Medicine, \\ Kaohsiung, 8330I, Taiwan
}

Purpose: We evaluate the association of Geriatric Nutritional Risk Index (GNRI) and the adverse outcome in elderly patients ( $\geq 65$ years old) with fall injuries.

Patients and Methods: Total 1071 elderly patients with fall injuries were enrolled. Patients were divided into four groups: high risk, moderate risk, low risk and no risk (GNRI: $<82,82$ to $<92,92$ to $\leq 98$ and $>98$ ) for patient demography, comorbidities, and adverse outcomes analysis.

Results: After 1:1 propensity score-matched analysis, 97 patients in high-risk group, 144 patients in moderate-risk group, and 114 patients in low-risk group were compared to no risk group. High-risk group patients had a 5.7-fold higher risk of mortality $(p=0.003)$ and prolong hospital stay (18.0 vs 12.3 days; $\mathrm{p}=0.016)$ when compared to no-risk group patients. Significantly prolong hospital stay were also found in low-risk and moderate-risk group when compared to no risk group.

Conclusion: A lower GNRI is associated with prolonged hospital stay in the elderly patients with fall injuries. High nutritional risk $($ GNRI $<82)$ is associated with an increased inhospital mortality rate.

Keywords: Geriatric Nutritional Risk Index, elderly patients, fall injuries, trauma

\section{Introduction}

Falls are the leading cause of trauma-related morbidity and mortality for the elderly. Approximately $5 \%$ to $10 \%$ of falls result in major head and orthopedic injuries, such as traumatic brain injuries and hip fractures, with mortality rates of $46 \%$ and $25 \%$, respectively. ${ }^{1,2}$ Falls are one of the major causes of hospital admission for traumatic injury among elderly patients. ${ }^{3}$ It has been reported that approximately $60 \%$ of patients are hospitalized due to injuries sustained during a fall. As the elderly population increases worldwide, traumatic injuries due to falls will, undoubtedly, increase as well.

The elderly are more likely to develop nutritional problems due to multiple comorbidities, such as altered gastrointestinal function, emotional distress, and polypharmacy. ${ }^{4,5}$ It is often acknowledged that providing adequate nutrition to the elderly may play a fundamental role in improving the skeletal muscles functioning. ${ }^{6}$ Lesser muscle strength is associated with a higher risk of falls, ${ }^{7-9}$ which may lead to adverse clinical events like prolonged length of hospital stay and in-hospital
Correspondence: Shih-Min Yin Department of General Surgery, Kaohsiung Chang Gung Memorial Hospital, No. 123, Dapi Road, Niaosong District, Kaohsiung City, 833, Taiwan, Republic of China Tel +886-9728-30436

Email dreamhunteryin@hotmail.com 
mortality. ${ }^{10,11}$ Therefore, it is crucial to have a timely diagnosis of malnutrition and a prompt initiation of proper nutritional care for elderly patients.

Recently, several assessment tools have been developed for the evaluation of nutritional status in clinical practice. The Geriatric Nutritional Risk Index (GNRI) was developed by Bouillanne et al in $2005 .^{12}$ It is a simplified and accurate screening tool, calculated using serum albumin level and a ratio of the patient's body weight to the ideal body weight (IBW). Lower GNRI is associated with higher degrees of malnutrition, inflammation, and sarcopenia. ${ }^{13}$ Several studies have also demonstrated that a low GNRI is a poor prognostic factor for certain malignancies and is associated with an increased risk of developing postoperative complications and an impaired survival rate. ${ }^{14-17}$ GNRI was also useful to identify the patients with malnutrition at a high risk of mortality among elderly patients with femoral fractures. ${ }^{18}$ However, the prognostic value and the clinical impact of the GNRI scoring in elderly patients with fall injuries have not been established yet.

The aim of this study was to evaluate the prognostic significance of the GNRI in estimating hospital mortality rate and the length of hospital stay for elderly patients with fall injuries.

\section{Patients and Methods Study Population}

This study was approved by the Institutional Review Board of the Kaohsiung Chang Gung Memorial Hospital, a Level I regional trauma center in southern Taiwan ${ }^{19,20}$ before the data collection was started (reference number 202001446B0). According to IRB regulations, the requirement for informed consent was waived due to the retrospective nature of this study. The privacy of the study subject and the confidentiality of the patient's information was compliance with the principles enunciated in the Declaration of Helsinki. This retrospective study reviewed the data of all the elderly ( $\geq 65$ years old) patients with fall injuries who were registered in the Trauma Registry System of the hospital from January 1, 2009 to December 31, 2019. Patients with incomplete data on body weight and albumin levels were excluded.

We applied the GNRI as an objective nutritional assessment tool for these elderly patients. The GNRI was calculated using the following equation: GNRI $=1.489 \times$ albumin $(\mathrm{g} / \mathrm{L})+[41.7 \times($ patient's body weight/IBW $)] .^{21}$
Based on the original paper by Bouillanne et al, ${ }^{12}$ IBW was calculated from estimated height $(\mathrm{H})$ and the Lorentz equations (WLo) as follows: $\mathrm{H}-100-[(\mathrm{H}-150) / 4]$ for men and $\mathrm{H}-100-[(\mathrm{H}-150) / 2.5]$ for women. Estimated height was calculated from knee height $(\mathrm{KH})$ and age by using the following equations taken from Chumlea et al: ${ }^{22}$ $[2.02 \mathrm{X} \mathrm{KH}(\mathrm{cm})]-[0.04 \mathrm{X}$ age $(\mathrm{y})]+64.19$ for men and $[1.83 \mathrm{X} \mathrm{KH}(\mathrm{cm})]-[0.24 \mathrm{X}$ age $(\mathrm{y})]+84.88$ for women. For patients with weight/IBW $\geq 1$, it was set to 1 according to the paper description from Bouillanne et al. ${ }^{12}$ The albumin level was check when patient was admitted to ward or intensive care unit (ICU). Patients were divided into four groups according to the grading of their nutritionrelated risk as derived from the GNRI values: high risk (GNRI: $<82$ ), moderate risk (GNRI: 82 to $<92$ ), low risk (GNRI: 92 to $\leqq 98$ ), and no risk (GNRI: $>98$ ) in line with the original paper description from Bouillanne et al. ${ }^{12}$

The detailed patient information, for this study, was retrieved from the Trauma Registry System of the hospital. The administrative databases that record medical conditions of the diseases were using International Classification of Diseases, 9th revision (ICD-9) and International Classification of Diseases, 10th revision (ICD-10). It included information on sex, age, body mass index (BMI), initial vital signs including body temperature (BT), heart rate (HR), respiratory rate (RR) and systolic blood pressure (SBP), blood transfusion $\geq 4$ units (U), comorbidities such as a history of cerebral vascular accidents (CVA), hypertension (HTN), coronary artery disease (CAD), congestive heart failure (CHF), diabetes mellitus (DM), and end-stage renal disease (ESRD); Glasgow coma scale (GCS) score and injury severity score (ISS). The detail definitions of comorbidities were using ICD-9 and ICD-10 (Supplement Table 1). ISS was expressed as a median with an interquartile range (IQR, Q1-Q3). In-hospital mortality and the length of hospital stay were also compared between the groups.

\section{Statistical Analysis}

The statistical analyses of the present study were performed using IBM SPSS Statistics for Windows, version 23.0 (IBM Corp., Armonk, NY, USA) and NCSS 10 software (NCSS Statistical Software, Kaysville, UT, USA). The primary outcomes of this study were in-hospital mortality and the length of hospital stay. Pearson chi-square tests were used to compare categorical data. The Kolmogorov-Smirnov test was used to analyze the normalization of the distributed data for continuous variables. Unpaired Student's t- and Post-Hoc Mann-Whitney $U$-tests were used to analyze normally 
distributed and non-normally distributed continuous data, respectively. To minimize the potential confounding effects due to the non-randomized assignment of the patients in this study, a logistic regression model, with sex, age, comorbidities, GCS score, ISS, initial vital signs and blood transfusion $>4 \mathrm{U}$ was used to calculate the propensity scores. We established a 1 to 1 propensity score-matched study group using the Greedy method with a 0.2 caliper width to compare the patients with no nutrition-related risk (GNRI > 98) with the patients from the other groups. The survival analyses were conducted using the Kaplan-Meier method with Log-rank tests. Odds ratios (ORs) with $95 \%$ confidence intervals (CIs) of the patients' condition between the groups were presented, and p-values $<0.05$ were defined as statistically significance.

\section{Results}

\section{Characteristics and Outcomes of the Elderly Patients with Different GNRIs}

As shown in Figure 1, 1071 elderly patients with fall injuries were included in the present study. According to the nutritionrelated risk, these patients were distributed into four groups: high risk $(\mathrm{N}=235$, GNRI < 82), moderate risk $(\mathrm{N}=371$, GNRI: 82 to <92), low-risk ( $\mathrm{N}=224$, GNRI: 92 to $<98$ ), and no risk $(\mathrm{N}=241, \mathrm{GNRI}>98)$. Table 1 shows that there were no significant differences in comorbidities, including CVA, HTN, CAD, CHF, DM and ESRD between the groups.
There were also no significant differences in initial BT, HR and RR. Compared to those of the patients with no nutritionrelated risk (GNRI > 98), patients with a lower GNRI in all the other groups had a significantly lower BMI, lower serum albumin level, and longer duration of hospital stay. In addition, patients in the high and moderate nutrition-related risk groups were significantly older, had lower initial SBP, prone to receive massive blood transfusion, and had higher mortality rates compared to that of patients with no nutrition-related risk. In the high nutrition-related risk group, a significantly lower number of patients had a GCS score in the 13-15 range and more patients had an ISS $>25$ when compared to that of patients in the no nutrition-related risk group.

\section{Adjusted Outcomes of the Propensity Score-Matched Patients}

After 1:1 propensity score-matched analysis, 97, 144, and 114 well-balanced pairs of patients were selected from the high-risk, the moderate-risk, and the low-risk groups, respectively. Compared to the no-risk group, either low-risk group, moderate risk group and highrisk group had no significant differences in sex, age, comorbidities, GCS score, and ISS. As demonstrated in Table 2, when compared to that of the no-risk group, the logistic regression analysis of these pairs of patients showed that the high-risk group had a 5.7-fold higher risk of mortality as calculated by the OR $(95 \%$ CI

Enrolled trauma patients (2009-2019)

$(\mathrm{n}=39,135)$

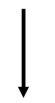

Elderly patients: age equal to or more than 65 years

$(\mathrm{n}=10,790)$

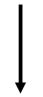

Fall

$(\mathrm{n}=6314)$

Excluded those that lack albumin or body weight data: $n=5243$

Study population

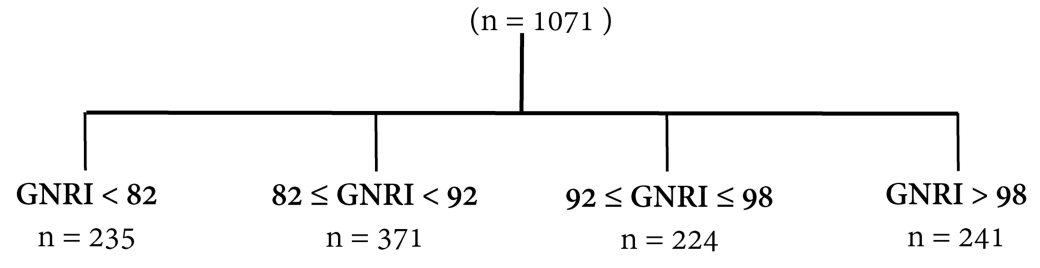

Figure I Flowchart on the elderly patients with fall injuries in different GNRI groups. 
Table I Relationships Between GNRI and Patient Demography in All Elderly Patients with Fall Injuries

\begin{tabular}{|c|c|c|c|c|c|}
\hline & \multicolumn{5}{|c|}{ GNRI } \\
\hline & $<82$ & 82 to $<92$ & 92 to $\leqq 98$ & $>98$ & \multirow[t]{2}{*}{$P$} \\
\hline & $n=235$ & $n=371$ & $n=224$ & $n=24 I$ & \\
\hline Gender & & & & & 0.004 \\
\hline Male & $109(46.4) *$ & $139(37.5)$ & $98(43.8) *$ & $76(31.5)$ & \\
\hline Female & $126(53.6) *$ & $232(62.5)$ & $126(56.2) *$ & $165(68.5)$ & \\
\hline Age & $81.1 \pm 7.4^{*}$ & $79.8 \pm 7.4^{*}$ & $78.4 \pm 7.1$ & $77.0 \pm 6.9$ & $<0.001$ \\
\hline BMI & $20.5 \pm 4.5^{*}$ & $22.8 \pm 3.9 *$ & $24.0 \pm 4.0^{*}$ & $25.3 \pm 3.5$ & $<0.001$ \\
\hline Albumin(g/dl) & $2.5 \pm 0.5^{*}$ & $3.1 \pm 0.3^{*}$ & $3.6 \pm 0.5^{*}$ & $4.1 \pm 0.2$ & $<0.001$ \\
\hline \multicolumn{6}{|l|}{ Co-Morbidity } \\
\hline CVA & $27(\mid 1.5)$ & $55(14.8)$ & $38(17.0)$ & $32(13.3)$ & 0.375 \\
\hline HTN & $134(57.0)$ & $247(66.6)$ & $144(64.3)$ & $164(68.0)$ & 0.052 \\
\hline CAD & $34(14.5)$ & $46(12.4)$ & $35(15.6)$ & $37(15.4)$ & 0.651 \\
\hline $\mathrm{CHF}$ & II (4.7) & $19(5.1)$ & $7(3.1)$ & $6(2.5)$ & 0.339 \\
\hline DM & $67(28.5)$ & $127(34.2)$ & $87(38.8)$ & $94(39.0)$ & 0.055 \\
\hline ESRD & $12(5.1)$ & $30(8.1)$ & $16(7.1)$ & $13(5.4)$ & 0.417 \\
\hline GCS, median (IQR) & $15(15-15) *$ & $15(15-15) *$ & $15(15-15)$ & $15(15-15)$ & 0.001 \\
\hline$\leqq 8$ & $19(8.1)$ & $30(8.1)$ & II(4.9) & II (4.6) & 0.187 \\
\hline $9-12$ & $18(7.7)$ & $34(9.2) *$ & $8(3.6)$ & $7(2.9)$ & 0.004 \\
\hline $13-15$ & $198(84.3) *$ & $307(82.7) *$ & $205(91.5)$ & $223(92.5)$ & $<0.001$ \\
\hline ISS, median (IQR) & $9(9-16)$ & $9(9-16)$ & $9(9-16)$ & $9(9-9)$ & $<0.001$ \\
\hline $1-15$ & $172(73.2)$ & $272(73.3)$ & $167(74.6)$ & $194(80.5)$ & 0.180 \\
\hline $16-24$ & $35(\mid 4.9)$ & $68(18.3)$ & $47(21.0)$ & $33(13.7)$ & 0.135 \\
\hline$\geq 25$ & $28(I 1.9) *$ & $3 I(8.4)$ & $10(4.5)$ & $14(5.8)$ & 0.014 \\
\hline Mortality & $50(21.3) *$ & $35(9.4) *$ & $10(4.5)$ & II (4.6) & $<0.001$ \\
\hline Hospital stay (days) & $21.2 \pm 19.8^{*}$ & $17.7 \pm 15.9 *$ & $14.7 \pm 1 \mid .7^{*}$ & $11.1 \pm 12.5$ & $<0.001$ \\
\hline BT & $36.6 \pm 0.7$ & $36.5 \pm 0.8$ & $36.6 \pm 0.6$ & $36.7 \pm 0.7$ & 0.240 \\
\hline$H R$ & $87.0 \pm 19.8$ & $86.6 \pm 18.0$ & $85.5 \pm 17.8$ & $83.6 \pm 17.1$ & 0.141 \\
\hline SBP & $151.5 \pm 37.6 *$ & $157.4 \pm 35.0 *$ & $157.2 \pm 37.1$ & $165.4 \pm 33.1$ & $<0.001$ \\
\hline $\mathrm{RR}$ & $18.5 \pm 2.3$ & $18.8 \pm 2.4$ & $18.7 \pm 2.0$ & $18.4 \pm 2.1$ & 0.054 \\
\hline Blood Transfusion & & & & & $<0.001$ \\
\hline$\geq 4 \mathrm{U}$ & $3 I(\mid 3.2)^{*}$ & $37(10.0) *$ & II(4.9) & $6(2.5)$ & \\
\hline$<4 U$ & $204(86.8)^{*}$ & $334(90.0)^{*}$ & $213(95.1)$ & $235(97.5)$ & \\
\hline
\end{tabular}

Notes: *Statistic significance comparing to no-risk (GNRI>98) group; the categorical data was compared with Pearson chi-square tests; the normally distributed and nonnormally distributed continuous data were analyzed by Unpaired Student's $t$ - and Post-Hoc Mann-Whitney U-tests, respectively. Significant values are indicated in Italic. Abbreviations: BMI, body mass index; CAD, coronary artery disease; CHF, congestive heart failure; $\mathrm{Cl}$, confidence interval; CVA, cerebral vascular accident; DM, diabetes mellitus; ESRD, end-stage renal disease; GCS, Glasgow Coma Scale; HTN, hypertension; IQR, interquartile range; ISS, injury severity score; BT, body temperature; HR, heart rate; SBP, systolic blood pressure; $R R$, respiratory rate; OR, odds ratio.

$1.60-20.50 ; p=0.003)$ and a significantly longer duration of hospital stay (18.0 days vs 12.3 days for the norisk group; $\mathrm{p}=0.016$ ). As shown in the Figure 2, the Kaplan-Meier curves for survival analysis also revealed inferior survival of high-risk group comparing to no risk group $(\mathrm{p}=0.016)$.
When the patients in the moderate and the low-risk groups were compared to those of the no-risk group (Tables 3 and 4), no significant differences in sex, age, comorbidities, GCS score, and ISS were found between the two patient cohorts. Compared with that of the no-risk group, the logistic regression analysis of these pairs of 
Table 2 Comparison Between High-Risk and No-Risk Group in Elderly Patients with Fall Injuries: Propensity Score Matched Analysis

\begin{tabular}{|c|c|c|c|c|c|}
\hline \multicolumn{6}{|c|}{ Propensity Score matched-cohort } \\
\hline & \multicolumn{5}{|c|}{ GNRI } \\
\hline & $\begin{array}{l}<82 \\
n=97\end{array}$ & $\begin{array}{l}>98 \\
n=97\end{array}$ & OR (95\%Cl) & $p$ & Standardized Difference \\
\hline \multicolumn{6}{|l|}{ Gender } \\
\hline Male & $35(36.1)$ & $35(36.1)$ & $1.0(0.56-1.80)$ & 1.000 & $0.00 \%$ \\
\hline Age & $80.1 \pm 7.3$ & $80.0 \pm 6.6$ & - & 0.876 & $2.24 \%$ \\
\hline \multicolumn{6}{|l|}{ Co-Morbidity } \\
\hline CVA & $2(2.1)$ & $2(2.1)$ & I.0(0.14-7.25) & 1.000 & $0.00 \%$ \\
\hline HTN & $64(66.0)$ & $64(66.0)$ & $\mathrm{I} .0(0.55-\mathrm{I} .8 \mathrm{I})$ & 1.000 & $0.00 \%$ \\
\hline CAD & $8(8.2)$ & $8(8.2)$ & I.0(0.36-2.78) & 1.000 & $0.00 \%$ \\
\hline $\mathrm{CHF}$ & $0(0.0)$ & $0(0.0)$ & - & - & - \\
\hline DM & $29(29.9)$ & $29(29.9)$ & $1.0(0.54-1.85)$ & 1.000 & $0.00 \%$ \\
\hline ESRD & $\mathrm{I}(\mathrm{I} .0)$ & $\mathrm{I}(\mathrm{I} .0)$ & $1.0(0.06-16.22)$ & 1.000 & $0.00 \%$ \\
\hline GCS, median (IQR) & $15(15-15)$ & $15(15-15)$ & & 0.614 & $-7.26 \%$ \\
\hline ISS, median (IQR) & $9(9-9)$ & $9(9-9)$ & - & 0.430 & $11.36 \%$ \\
\hline BT & $36.6 \pm 0.7$ & $36.6 \pm 0.6$ & - & 0.913 & $-1.56 \%$ \\
\hline$H R$ & $82.7 \pm 15.6$ & $84.2 \pm 15.2$ & - & 0.488 & $-9.99 \%$ \\
\hline SBP & $160.3 \pm 33.5$ & $158.3 \pm 27.8$ & - & 0.661 & $6.30 \%$ \\
\hline $\mathrm{RR}$ & $18.5 \pm 2.0$ & $18.6 \pm 1.5$ & - & 0.773 & $-4.15 \%$ \\
\hline Blood Transfusion & $0(0.0)$ & $0(0.0)$ & - & - & \\
\hline \multicolumn{6}{|l|}{$\geq 4 \cup$} \\
\hline Mortality & $15(\mid 5.5)$ & $3(3.1)$ & $5.7(1.60-20.50)$ & 0.003 & - \\
\hline Hospital stay (days) & $18.0 \pm 15.9$ & $12.3 \pm 16.8$ & - & 0.016 & - \\
\hline
\end{tabular}

Notes: The categorical data was compared with Pearson chi-square tests; The normally distributed and non-normally distributed continuous data were analyzed by Unpaired Student's $t$ - and Post-Hoc Mann-Whitney U-tests, respectively. Significant values are indicated in Italic.

Abbreviations: BMI, body mass index; CAD, coronary artery disease; CHF, congestive heart failure; $\mathrm{Cl}$, confidence interval; CVA, cerebral vascular accident; DM, diabetes mellitus; ESRD, end-stage renal disease; GCS, Glasgow Coma Scale; HTN, hypertension; IQR, interquartile range; ISS, injury severity score; BT, body temperature; HR, heart rate; SBP, systolic blood pressure; RR, respiratory rate; OR, odds ratio.

patients showed a significant difference in the odds of staying at the hospital for longer durations for both the moderate-risk group (17.0 days vs 11.3 days for the norisk group; $p=0.002$ ) and the low-risk group (13.1 days vs 10.3 days for the no-risk group; $\mathrm{p}=0.04)$. When compared with the no-risk group of patients, the patients in the moderate and the low-risk groups did not have significant different odds of mortality from propensity-score matched populations and did not present significant difference of survival curves in the Kaplan-Meier analysis.

\section{Discussion}

Among the elderly population, malnutrition is a critical and common issue and is usually related to a poor prognosis in the patients with many comorbidities, ${ }^{23-25}$ like cardiovascular disease and various malignancies. In the present study, we demonstrated the relationship between the nutritional status assessed by the GNRI and adverse prognosis in the elderly patients with fall injuries.
Compared to that of the patients of the no-risk group (GNRI > 98), the in-hospital mortality rate in patients with moderate risk (GNRI 82 to $\leqq 92$ ) and high risk (GNRI < 82) was significantly higher. Patients with a low GNRI also had a longer hospital stay than that of patients in the no-risk group. Notably, after adjustment for the differences in the demographic characteristics, comorbidities, initial vital signs, blood transfusion $\geq 4 \mathrm{U}$ and injury severity of the trauma patients, patient in the low, moderate, and high-risk groups still had significantly prolonged hospital stay when compared to those of patients in the no-risk group. Additionally, we reported that a GNRI of $<82$ is an independent predictor of in-hospital mortality and inferior survival in elderly patients with fall injuries.

Malnutrition status may contribute to increased risk of inferior clinical outcome in trauma patients through several mechanisms. Deficiency of calorie and protein is associated with osteoporosis and sarcopenia, which may reduce the bone resistance to trauma and increases the risk 

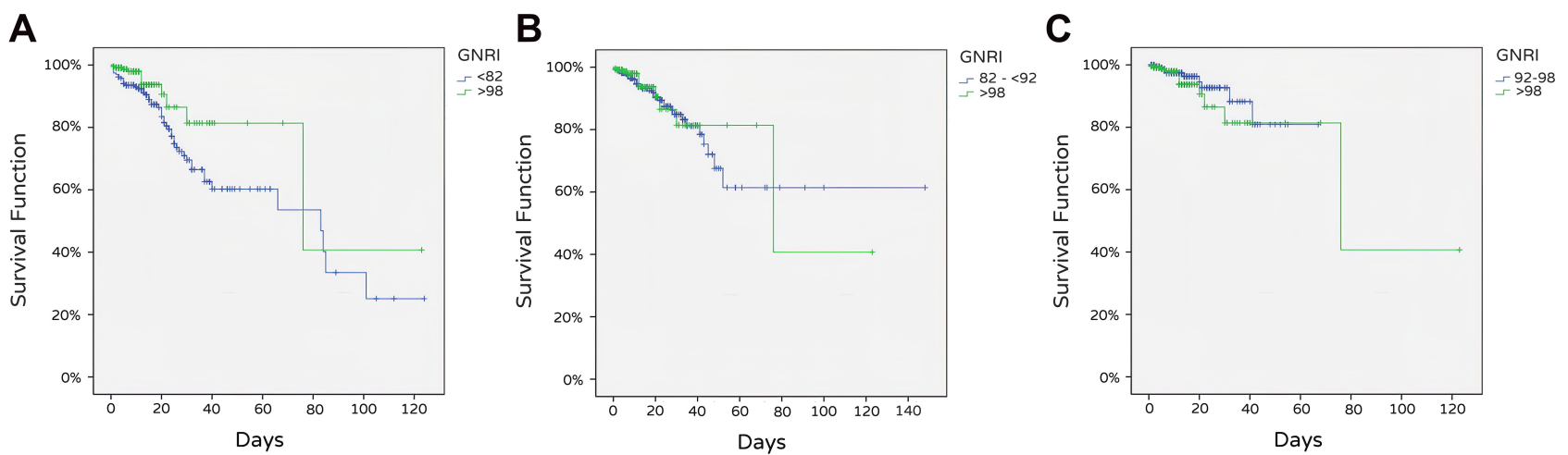

Figure 2 Kaplan-Meier curves based on GNRI in according to different nutritional risk in patients with fall injury. (A) high-risk group and no-risk group, Log-Rank $\mathrm{P}=0.0 \mathrm{I}$; (B) moderate-risk group and no-risk group, Log-Rank $\mathrm{P}=0.662$; (C) low-risk group and no-risk group, Log-Rank $\mathrm{P}=0.453$.

of falls. ${ }^{26}$ Previous studies also reported that higher protein and adequate energy intake was associated with less postoperative complications, as well as decreased duration of hospital stay. ${ }^{27,28}$ Since the ratio of the present to the ideal body weight can reflect the caloric depletion and serum albumin may reflect protein reservation, ${ }^{29}$ the malnutrition defined by low GNRI is a potential indicator for the associated adverse outcome following the trauma.

Table 3 Comparison Between Moderate-Risk and No-Risk Group in Elderly Patients with Fall Injuries: Propensity Score Matched Analysis

\begin{tabular}{|c|c|c|c|c|c|}
\hline \multicolumn{6}{|c|}{ Propensity Score matched-cohort } \\
\hline & \multicolumn{5}{|c|}{ GNRI } \\
\hline & $\begin{array}{l}82 \text { to }<92 \\
n=144\end{array}$ & $\begin{array}{l}>98 \\
n=144\end{array}$ & OR $(95 \% \mathrm{Cl})$ & $p$ & Standardized Difference \\
\hline Gender & & & & & \\
\hline Male & $43(29.9)$ & $43(29.9)$ & $1.0(0.60-1.66)$ & 1.000 & $0.00 \%$ \\
\hline Age & $78.8 \pm 6.5$ & $78.0 \pm 6.7$ & - & 0.357 & $10.88 \%$ \\
\hline Co-Morbidity & & & & & \\
\hline CVA & $12(8.3)$ & $12(8.3)$ & $\mathrm{I} .0(0.43-2.3 \mathrm{I})$ & 1.000 & $0.00 \%$ \\
\hline HTN & $98(68.1)$ & $98(68.1)$ & $1.0(0.6 \mathrm{I}-\mathrm{I} .64)$ & 1.000 & $0.00 \%$ \\
\hline CAD & $13(9.0)$ & I3(9.0) & I.0(0.45-2.24) & 1.000 & $0.00 \%$ \\
\hline $\mathrm{CHF}$ & $\mathrm{I}(0.7)$ & $\mathrm{I}(0.7)$ & $1.0(0.06-16.14)$ & 1.000 & $0.00 \%$ \\
\hline DM & $55(38.2)$ & $55(38.2)$ & $1.0(0.62-1.6 I)$ & 1.000 & $0.00 \%$ \\
\hline ESRD & $2(1.4)$ & $2(1.4)$ & I.0(0.14-7.20) & 1.000 & $0.00 \%$ \\
\hline GCS, median (IQR) & $15(15-15)$ & $15(15-15)$ & - & 0.209 & $14.85 \%$ \\
\hline ISS, median (IQR) & $9(9-9)$ & $9(9-9)$ & - & 0.252 & $-13.54 \%$ \\
\hline BT & $36.6 \pm 0.7$ & $36.7 \pm 0.7$ & - & 0.436 & $-9.18 \%$ \\
\hline$H R$ & $83.1 \pm 15.2$ & $85.8 \pm 15.4$ & - & 0.136 & $-17.64 \%$ \\
\hline SBP & $163.7 \pm 29.8$ & $163.1 \pm 30.0$ & - & 0.866 & $2.00 \%$ \\
\hline $\mathrm{RR}$ & $18.5 \pm 1.8$ & $18.5 \pm 1.6$ & - & 0.837 & $2.42 \%$ \\
\hline $\begin{array}{l}\text { Blood Transfusion } \\
\geq 4 \mathrm{U}\end{array}$ & $2(1.4)$ & $2(1.4)$ & $1.0(0.14-7.20)$ & 1.000 & $0.00 \%$ \\
\hline Mortality & $8(5.6)$ & $3(2.1)$ & $2.8(0.72-10.64)$ & 0.124 & - \\
\hline Hospital stay (days) & $17.0 \pm 17.5$ & $11.3 \pm 13.7$ & - & 0.002 & - \\
\hline
\end{tabular}

Notes: The categorical data was compared with Pearson chi-square tests; The normally distributed and non-normally distributed continuous data were analyzed by Unpaired Student's $t$ - and Post-Hoc Mann-Whitney U-tests, respectively. Significant values are indicated in Italic.

Abbreviations: BMI, body mass index; CAD, coronary artery disease; CHF, congestive heart failure; Cl, confidence interval; CVA, cerebral vascular accident; DM, diabetes mellitus; ESRD, end-stage renal disease; GCS, Glasgow Coma Scale; HTN, hypertension; IQR, interquartile range; ISS, injury severity score; BT, body temperature; HR, heart rate; SBP, systolic blood pressure; $R R$, respiratory rate; OR, odds ratio. 
Table 4 Comparison Between Low-Risk and No-Risk Group in Elderly Patients with Fall Injuries: Propensity Score Matched Analysis

\begin{tabular}{|c|c|c|c|c|c|}
\hline \multicolumn{6}{|c|}{ Propensity Score matched-cohort } \\
\hline & \multicolumn{5}{|c|}{ GNRI } \\
\hline & $\begin{array}{l}92 \text { to } \leqq 98 \\
n=|| 4\end{array}$ & $\begin{array}{l}>98 \\
n=114\end{array}$ & OR $(95 \% \mathrm{Cl})$ & $p$ & $\begin{array}{c}\text { Standardized } \\
\text { Difference }\end{array}$ \\
\hline Gender & & & & & \\
\hline Male & $38(33.3)$ & $38(33.3)$ & I.0(0.58-I.73) & 1.000 & $0.00 \%$ \\
\hline Age & $78.3 \pm 7.3$ & $78.4 \pm 6.6$ & - & 0.886 & $-1.90 \%$ \\
\hline Co-Morbidity & & & & & \\
\hline CVA & $8(7.0)$ & $8(7.0)$ & $1.0(0.36-2.76)$ & 1.000 & $0.00 \%$ \\
\hline HTN & $76(66.7)$ & $76(66.7)$ & $1.0(0.58-1.73)$ & 1.000 & $0.00 \%$ \\
\hline CAD & $9(7.9)$ & $9(7.9)$ & $1.0(0.38-2.62)$ & 1.000 & $0.00 \%$ \\
\hline $\mathrm{CHF}$ & $\mathrm{I}(0.9)$ & $\mathrm{I}(0.9)$ & $1.0(0.06-16.18)$ & 1.000 & $0.00 \%$ \\
\hline DM & $40(35.1)$ & $40(35.1)$ & $1.0(0.58-1.72)$ & 1.000 & $0.00 \%$ \\
\hline ESRD & $3(2.6)$ & $3(2.6)$ & $1.0(0.20-5.06)$ & 1.000 & $0.00 \%$ \\
\hline GCS, median (IQR) & $15(15-15)$ & $15(15-15)$ & - & 0.578 & $-7.38 \%$ \\
\hline ISS, median (IQR) & $9(9-9)$ & $9(9-9)$ & - & 0.705 & $5.02 \%$ \\
\hline BT & $36.6 \pm 0.7$ & $36.6 \pm 005$ & - & 0.682 & $5.44 \%$ \\
\hline$H R$ & $82.9 \pm 13.7$ & $84.0 \pm 15.5$ & - & 0.574 & $-7.45 \%$ \\
\hline SBP & $158.6 \pm 32.9$ & $162.3 \pm 28.7$ & - & 0.374 & $-11.79 \%$ \\
\hline $\mathrm{RR}$ & 18. $5 \pm 2.1$ & $18.6 \pm 1.5$ & - & 0.684 & $-5.39 \%$ \\
\hline $\begin{array}{l}\text { Blood Transfusion } \\
\geq 4 \cup\end{array}$ & $0(0.0)$ & $0(0.0)$ & - & - & - \\
\hline Mortality & $3(105)$ & $3(2.6)$ & $1.0(0.20-5.06)$ & 1.000 & - \\
\hline Hospital stay(day) & $13.1 \pm 10.4$ & $10.3 \pm 9.8$ & - & 0.040 & - \\
\hline
\end{tabular}

Notes: The categorical data was compared with Pearson chi-square tests; The normally distributed and non-normally distributed continuous data were analyzed by Unpaired Student's $t$ - and Post-Hoc Mann-Whitney U-tests, respectively. Significant values are indicated in Italic.

Abbreviations: BMI, body mass index; CAD, coronary artery disease; CHF, congestive heart failure; $\mathrm{Cl}$, confidence interval; CVA, cerebral vascular accident; DM, diabetes mellitus; ESRD, end-stage renal disease; GCS, Glasgow Coma Scale; HTN, hypertension; IQR, interquartile range; ISS, injury severity score; BT, body temperature; HR, heart rate; SBP, systolic blood pressure; RR, respiratory rate; OR, odds ratio.

Several nutritional assessment tools have been developed previously, like BMI, skeletal muscle mass index, prognostic nutritional index, ${ }^{30}$ and subjective global assessment. ${ }^{31}$ However, the clinical applicability of these tools for the elderly needs to be elucidated as there is a lack of consensus regarding their clinical utility. Many age-related medical conditions, such as cognitive dysfunction, visual impairment, depressed mood, increase in the adverse effects of drugs due to polypharmacy, and a low muscle mass and strength, may lead to an increased risk of injuries due to falls in the elderly. ${ }^{7,32}$ However, the evaluation of the malnutrition status for these patients with fall injuries is not fully standardized. However, the GNRI has been reported as a useful and efficient screening tool for the nutritional status during the overall evaluation of the hospitalized elderly patients. The GNRI is calculated using body weight, height, and serum albumin level, which can be accurately and easily evaluated during admission.

In some previous studies, the GNRI has been reported as a prognostic predictor for patients undergoing hemodialysis and those suffering from many acute and chronic conditions, such as acute respiratory distress syndrome and heart failure. ${ }^{33,34}$ Recently, the GNRI was also considered as a useful prognostic indicator of morbidity and mortality in patients with gastrointestinal cancer, as well as overall survival. ${ }^{35,36}$ However, the literature on the application of the GNRI as a prognostic predictor for the elderly patients suffering from traumatic injuries is very limited. Our study confirmed that a lower GNRI may be associated with a longer hospital stay, even in the moderate-and the low-risk groups. If the patient is severely malnourished $($ GNRI $<82)$ when a traumatic injury due 
to fall occurs, it may also result in an increased in-hospital mortality. Therefore, we recommend that for an elderly patient with a low GNRI, who has sustained a fall injury, the physicians should promptly initiate the appropriate treatment, and should also consider more aggressive medical care.

Several limitations should be considered when interpreting the results of the current study. First, the selection bias due to the retrospective study design could not be completely excluded; even after applying the propensity score matching method. A large proportion of patients with falls did not have serum albumin data at admission and such scenario may lead to a selection bias. However, this also reflects that the assessment of the nutritional status is often neglected even for the patients with fall. Second, although the severity of trauma was evaluated using ISS, the causes of mortality and longer hospital stay may vary due to the differences in the severity of trauma and for different comorbidities. Third, we only used a baseline GNRI measurement for the nutritional status evaluation in this study. Although there are other nutritional assessment tools, such as the muscle power and muscle mass status, we were not able to evaluate and compare these tools with the GNRI due to insufficient data. Further studies might need to employ more nutritional and physical evaluation assessment tools to understand the association between malnutrition and fall injuries in the elderly patients.

\section{Conclusions}

In conclusion, a lower GNRI is associated with adverse outcomes as prolonged hospital stay in the elderly patients with fall injuries. High nutritional risk $($ GNRI $<82)$ is associated with an increased in-hospital mortality rate. Physicians should consider more intensive clinical observation and treatment to improve the prognosis of the elderly patients who suffered from fall injuries and have a poor GNRI status.

\section{Acknowledgments}

We would like to extend our gratitude to the Biostatistics Center of Kaohsiung Chang Gung Memorial Hospital for their assistance with statistical analyses.

\section{Author Contributions}

All authors contributed to data analysis, drafting or revising the article, have agreed on the journal to which the article will be submitted, gave final approval of the version to be published, and agree to be accountable for all aspects of the work. Szu-Wei Huang wrote the manuscript, SzuWei Huang, Shih-Min Yin was involved in the literature review, Ching-Hua Hsieh performed the statistical analyses, Shih-Min Yin, Ching-Hua Hsieh proofread the manuscript, Szu-Wei Huang was responsible for the integrity of registered data, and Ching-Hua Hsieh designed the study and contributed to the data analysis and interpretation.

\section{Disclosure}

The authors declare no conflicts of interest.

\section{References}

1. Goldacre MJ, Roberts SE, Yeates D. Mortality after admission to hospital with fractured neck of femur: database study. BMJ. 2002;325 (7369):868-869. doi:10.1136/bmj.325.7369.868

2. Murphy DK, Randell T, Brennan KL, Probe RA, Brennan ML. Treatment and displacement affect the reoperation rate for femoral neck fracture. Clin Orthop Relat Res. 2013;471(8):2691-2702. doi:10.1007/s11999-013-3020-9

3. Rau CS, Lin TS, Wu SC, et al. Geriatric hospitalizations in fall-related injuries. Scand J Trauma Resusc Emerg Med. 2014;22:63. doi:10.1186/s13049-014-0063-1

4. Vellas B, Lauque S, Andrieu S, et al. Nutrition assessment in the elderly. Curr Opin Clin Nutr Metab Care. 2001;4(1):5-8. doi:10.1097/00075197-200101000-00002

5. Oliveira MR, Fogaca KC, Leandro-Merhi VA. Nutritional status and functional capacity of hospitalized elderly. Nutr J. 2009;8:54. doi:10.1186/1475-2891-8-54

6. Romagnoli C, Pampaloni B, Brandi ML. Muscle endocrinology and its relation with nutrition. Aging Clin Exp Res. 2019;31(6):783-792. doi:10.1007/s40520-019-01188-5

7. Moreland JD, Richardson JA, Goldsmith CH, Clase CM. Muscle weakness and falls in older adults: a systematic review and meta-analysis. $J$ Am Geriatr Soc. 2004;52(7):1121-1129. doi:10.1111/j.1532-5415.2004.52310.x

8. Pijnappels M, van der Burg PJ, Reeves ND, van Dieen JH. Identification of elderly fallers by muscle strength measures. Eur $J$ Appl Physiol. 2008;102(5):585-592. doi:10.1007/s00421-0070613-6

9. Clynes MA, Edwards MH, Buehring B, Dennison EM, Binkley N, Cooper C. Definitions of sarcopenia: associations with previous falls and fracture in a population sample. Calcif Tissue Int. 2015;97 (5):445-452. doi:10.1007/s00223-015-0044-z

10. Vetrano DL, Landi F, Volpato S, et al. Association of sarcopenia with short- and long-term mortality in older adults admitted to acute care wards: results from the CRIME study. J Gerontol a Biol Sci Med Sci. 2014;69(9):1154-1161. doi:10.1093/gerona/glu034

11. Peterson SJ, Braunschweig CA. Prevalence of sarcopenia and associated outcomes in the clinical setting. Nutr Clin Pract. 2016;31 (1):40-48. doi:10.1177/0884533615622537

12. Bouillanne O, Morineau G, Dupont C, et al. Geriatric nutritional risk index: a new index for evaluating at-risk elderly medical patients. $\mathrm{Am}$ J Clin Nutr. 2005;82(4):777-783. doi:10.1093/ajcn/82.4.777

13. Kuo IC, Huang JC, Wu PY, Chen SC, Chang JM, Chen HC. A low geriatric nutrition risk index is associated with progression to dialysis in patients with chronic kidney disease. Nutrients. 2017;9(11):1228. doi:10.3390/nu9111228

14. Lidoriki I, Schizas D, Frountzas M, et al. GNRI as a prognostic factor for outcomes in cancer patients: a systematic review of the literature. Nutr Cancer. 2021;73:391-403. 
15. Balzano G, Dugnani E, Crippa S, et al. A preoperative score to predict early death after pancreatic cancer resection. Dig Liver Dis. 2017;49(9):1050-1056. doi:10.1016/j.dld.2017.06.012

16. Kushiyama S, Sakurai K, Kubo N, et al. The preoperative geriatric nutritional risk index predicts postoperative complications in elderly patients with gastric cancer undergoing gastrectomy. In Vivo. 2018;32 (6):1667-1672. doi:10.21873/invivo. 11430

17. Yamana I, Takeno S, Shimaoka H, et al. Geriatric nutritional risk index as a prognostic factor in patients with esophageal squamous cell carcinoma -retrospective cohort study. Int J Surg. 2018;56:44-48. doi:10.1016/j.ijsu.2018.03.052

18. Su WT, Wu SC, Huang CY, et al. Geriatric nutritional risk index as a screening tool to identify patients with malnutrition at a high risk of in-hospital mortality among elderly patients with femoral fractures-a retrospective study in a level I trauma center. Int J Environ Res Public Health. 2020;17(23):8920. doi:10.3390/ ijerph 17238920

19. Hsieh CH, Hsu SY, Hsieh HY, Chen YC. Differences between the sexes in motorcycle-related injuries and fatalities at a Taiwanese level I trauma center. Biomed J. 2017;40(2):113-120. doi:10.1016/j. bj.2016.10.005

20. Hsieh CH, Liu HT, Hsu SY, Hsieh HY, Chen YC. Motorcycle-related hospitalizations of the elderly. Biomed J. 2017;40(2):121-128. doi:10.1016/j.bj.2016.10.006

21. Pablo AM, Izaga MA, Alday LA. Assessment of nutritional status on hospital admission: nutritional scores. Eur J Clin Nutr. 2003;57 (7):824-831. doi:10.1038/sj.ejcn.1601616

22. Chumlea WC, Roche AF, Steinbaugh ML. Estimating stature from knee height for persons 60 to 90 years of age. J Am Geriatr Soc. 1985;33(2):116-120. doi:10.1111/j.1532-5415.1985.tb02276.x

23. Zheng HL, Lu J, Li P, et al. Effects of preoperative malnutrition on short- and long-term outcomes of patients with gastric cancer: can we do better? Ann Surg Oncol. 2017;24(11):3376-3385. doi:10.1245/ s10434-017-5998-9

24. $\mathrm{Xu}$ J, Zhou $\mathrm{X}$, Zheng $\mathrm{C}$. The geriatric nutritional risk index independently predicts adverse outcomes in patients with pyogenic liver abscess. BMC Geriatr. 2019;19(1):14. doi:10.1186/s12877-019-1030-5

25. Zhao Q, Zhang TY, Cheng YJ, et al. Impacts of geriatric nutritional risk index on prognosis of patients with non-ST-segment elevation acute coronary syndrome: results from an observational cohort study in China. Nutr Metab Cardiovasc Dis. 2020;30(10):1685-1696. doi:10.1016/j.numecd.2020.05.016
26. Huang Z, Himes JH, McGovern PG. Nutrition and subsequent hip fracture risk among a national cohort of white women. Am J Epidemiol. 1996;144(2):124-134. doi:10.1093/oxfordjournals.aje. a008899

27. Botella-Carretero JI, Iglesias B, Balsa JA, Arrieta F, Zamarron I, Vazquez C. Perioperative oral nutritional supplements in normally or mildly undernourished geriatric patients submitted to surgery for hip fracture: a randomized clinical trial. Clin Nutr. 2010;29 (5):574-579

28. Anbar R, Beloosesky Y, Cohen J, et al. Tight calorie control in geriatric patients following hip fracture decreases complications: a randomized, controlled study. Clin Nutr. 2014;33(1):23-28. doi:10.1016/j.clnu.2013.03.005

29. Hirahara N, Matsubara T, Hayashi H, Takai K, Nakada S, Tajima Y. Prognostic importance of controlling nutritional status in patients undergoing curative thoracoscopic esophagectomy for esophageal cancer. $\mathrm{Am}$ J Ther. 2018;25(5):e524-e532. doi:10.1097/MJT.0000000000000414

30. Buzby GP, Mullen JL, Matthews DC, Hobbs CL, Rosato EF. Prognostic nutritional index in gastrointestinal surgery. Am J Surg. 1980;139(1):160-167. doi:10.1016/0002-9610(80)90246-9

31. Detsky AS, McLaughlin JR, Baker JP, et al. What is subjective global assessment of nutritional status? JPEN J Parenter Enteral Nutr. 1987;11(1):8-13. doi:10.1177/014860718701100108

32. O’Neill S, Brady RR, Kerssens JJ, Parks RW. Mortality associated with traumatic injuries in the elderly: a population based study. Arch Gerontol Geriatr. 2012;54(3):e426-430. doi:10.1016/j.archger.2012.01.007

33. Kaneko H, Suzuki S, Goto M, et al. Geriatric nutritional risk index in hospitalized heart failure patients. Int J Cardiol. 2015;181:213-215. doi:10.1016/j.ijcard.2014.11.167

34. Yoo JW, Ju S, Lee SJ, Cho YJ, Lee JD, Kim HC. Geriatric nutritional risk index is associated with 30-day mortality in patients with acute respiratory distress syndrome. Medicine. 2020;99(25):e20671. doi:10.1097/MD.0000000000020671

35. Hirahara N, Matsubara T, Fujii Y, et al. Preoperative geriatric nutritional risk index is a useful prognostic indicator in elderly patients with gastric cancer. Oncotarget. 2020;11(24):2345-2356. doi:10.18632/oncotarget.27635

36. Sasaki M, Miyoshi N, Fujino S, et al. The Geriatric Nutritional Risk Index predicts postoperative complications and prognosis in elderly patients with colorectal cancer after curative surgery. Sci Rep. 2020;10(1):10744. doi:10.1038/s41598-020-67285-y
Risk Management and Healthcare Policy

\section{Publish your work in this journal}

Risk Management and Healthcare Policy is an international, peerreviewed, open access journal focusing on all aspects of public health, policy, and preventative measures to promote good health and improve morbidity and mortality in the population. The journal welcomes submitted papers covering original research, basic science, clinical \& epidemiological studies, reviews and evaluations, guidelines, expert opinion and commentary, case reports and extended reports. The manuscript management system is completely online and includes a very quick and fair peer-review system, which is all easy to use. Visit http://www.dovepress.com/testimonials.php to read real quotes from published authors. 